\title{
Prophylactic inguinal-femoral irradiation as an alternative to primary lymphadenectomy in treatment of vulvar carcinoma
}

\author{
SORANA HALLAK ${ }^{1}$, LUZ LADI $^{2}$ and BENGT SORBE ${ }^{1}$ \\ ${ }^{1}$ Department of Gynecological Oncology, and ${ }^{2}$ Department of Obstetrics and Gynecology, \\ Örebro University Hospital, S-701 85 Örebro, Sweden
}

Received January 8, 2007; Accepted June 7, 2007

\begin{abstract}
In a complete geographic series of 294 cases of primary vulvar carcinomas prophylactic inguinal-femoral irradiation was used as a standard postoperative therapy. Inguinal lymph node dissection was performed in only 27 cases $(9 \%)$ and was not part of the standard surgery. The histology was squamous cell carcinoma in 269 cases (92\%). The primary surgery was total vulvectomy, partial vulvectomy, or local resection of the tumor. The main type of radiotherapy was adjuvant inguinal irradiation. Two separate, symmetrical and rectangular inguinal fields were irradiated with combined photon and electron beams. In the complete series 127 recurrences $(43 \%)$ were recorded. Local $(24 \%)$ and regional recurrences $(19 \%)$ were most frequent. Type of surgery was not associated with the risk of tumor recurrence. The 5-year overall survival rate was $53 \%$ and the relapse-free survival (RFS) rate was $55 \%$. Tumor grade was significantly $(\mathrm{P}=0.007)$ associated with the RFS. The inguinal RFS rate was $75 \%$ both for patients treated with adjuvant inguinal irradiation without lymphadenectomy and patients treated with primary lymphadenectomy \pm inguinal irradiation. Postoperative complications were recorded in $22 \%$. Postoperative complications occurred most frequently in the subgroup undergoing inguinal lymphadenectomy. Chronic lymph edemas were the most serious late tissue reactions.
\end{abstract}

\section{Introduction}

Cancer of the vulva is uncommon and represents $4 \%$ of all gynecological malignancies (1). The mean age of the patients is 70 years. Squamous cell carcinomas account for $90 \%$ of the cases (2). The overall prognosis for all stages of primary squamous cell carcinomas of the vulva is favorable and the 5-year survival rate is reported to be as high as $70 \%(3,4)$. Approximately $40 \%$ of the patients with vulvar cancer present

Correspondence to: Professor Bengt Sorbe, Department of Gynecological Oncology, Örebro University Hospital, S-701 85 Örebro, Sweden

E-mail: bengt.sorbe@orebroll.se

Key words: vulvar carcinoma, inguinal-femoral irradiation, lymphadenectomy, prognosis with advanced disease (FIGO stages III and IV) at diagnosis. In advanced stages $45 \%$ of the tumors will recur and the median disease-free survival is then only 35 months.

Lymph node metastasis is the single most important prognostic factor for survival; the presence of inguinal lymph node metastases results in a $50 \%$ reduction in long-term survival (5). The salvage rate for patients with groin recurrence is less than $10 \%$. The median time to recurrence in the groin is 7 months. Most recurrences are diagnosed within 2 years. The median survival after recurrence in the groin is 9 months (6). Only $20-30 \%$ of the patients in early stages have positive inguinal-femoral lymph node metastases. The prognosis is better after lymphadenectomy compared to surveillance of the groin (2). The results of palpation in predicting the lymph node status are disappointing (7). In a prospective GOG study the involvement of inguinal lymph nodes was rated as clinically negative in $81 \%$ of the cases. Of the clinically negative lymph nodes, $24 \%$ were affected histopathological by tumor. A false-positive lymph node diagnosis was obtained in $20 \%$ of the cases (8).

If palpable inguinal lymph nodes are present, a lymphadenectomy is preferred, followed by irradiation to $50 \mathrm{~Gy}$ to the inguinal-femoral and pelvic nodes. If irradiation alone is used, additional doses are delivered after 45 Gy with reduced fields to complete 65-70 Gy (9). Some investigators have suggested treating patients with clinically negative groins with wide local excision of the vulva combined with radiotherapy of the groin only. The use of elective groin irradiation in patients with vulvar cancer is controversial (2). Irradiation of $\mathrm{N} 0$ or $\mathrm{N} 1$ inguinal-femoral nodes is an alternative to lymphadenectomy for squamous cell carcinoma of the vulva if proper irradiation technique and dose are used. The acute and late morbidity are less than with lymphadenectomy (10). In 1992, Stehman et al published GOG study 88 (6). The objective of that study was to document the rates of recurrence between patients randomly assigned to either groin dissection or radiation to the intact groin. Five out of 27 patients (18.5\%) who received primary groin radiotherapy developed a groin recurrence, which is an unacceptably high failure rate, and therefore the study was closed far in advance. A Cochrane review in 2002 stated that primary radiotherapy to the groin results in less morbidity but in a higher number of groin recurrences compared with surgery (11). A GOG study showed that radical surgical intervention resulted in chronic lymphatic edema of the lower extremity in $27 \%$ of the cases (12). 
Table I. Tumor characteristics of the complete series.

\begin{tabular}{lrr}
\hline & Number & Percent \\
\hline Stage & 110 & 37.4 \\
I & 115 & 39.1 \\
II & 43 & 14.6 \\
III & 26 & 8.9 \\
IV & & \\
Histology & 269 & 91.5 \\
Squamous cell carcinoma & 10 & 3.4 \\
Malignant melanoma & 8 & 2.7 \\
Paget's disease of the vulva & 7 & 2.4 \\
Other types & & \\
Tumor grade & & \\
Well differentiated & 117 & 39.8 \\
Moderately differentiated & 123 & 41.8 \\
Poorly differentiated & 31 & 10.5 \\
Not graded & 23 & 7.8 \\
\hline
\end{tabular}

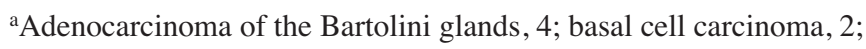
sarcoma, 1 .

Omission of groin radiation seems to be justified in low risk cases in the T1, N0-1 stage (no central location, no vessel invasion, tumor thickness $\leq 2 \mathrm{~mm}$, and G1 and G2 tumors) (4).

The trend in surgical management for women with invasive vulvar carcinoma over the past 30 years has been increasingly more conservative, with surgeons seeking to reduce morbidity while improving survival (13). Present treatment involves wide local excision with unilateral or bilateral inguinal-femoral lymphadenectomy via the triple incision technique. Postoperative radiotherapy is given in the case of more than one intranodal metastasis or one lymph node metastasis with extra nodal growth (2). Hacker et al showed that survival with the triple incision technique was similar to that in historical controls that had en block radical surgery (14). Shimm et al did not find a relationship between the extent of surgery and survival or local control. It is likely that involvement of pelvic lymph nodes indicates systemic dissemination of carcinoma, and is unlikely to respond favorably to locally directed adjuvant treatment (15). The tumor-free margins are the most important predictive factor for local recurrence rate $(2,16)$. De Hullu et al showed no local recurrence if margins were $>8 \mathrm{~mm}$, but $22.5 \%$ if margins were $<8 \mathrm{~mm}$ (2). Therefore, the authors advise wide local excision with an intentional surgical margin of $2 \mathrm{~cm}$.

The identification rate of the sentinel node technique is nearly $100 \%$ with preoperative lymphoscintigram and probe guided surgery. The blue technique gives a lower detection rate; $56 \%$ according to Ansink et al (multicenter study, beginning of the learning curve) (17).

In conclusion, prospective randomized studies are necessary to determine whether primary radiotherapy with an adequate radiation dose and technique is a safe treatment of the groin in a selection of patients with early stage vulvar cancer (2).
Table II. Surgical technique used in the complete series.

\begin{tabular}{lrr}
\hline & Number & Percent \\
\hline Type of surgery & & \\
Radical vulvectomy & 165 & 56.1 \\
Partial vulvectomy & 63 & 21.4 \\
Local excision & 43 & 14.6 \\
Multiple px & 23 & 7.8 \\
Surgical method & & \\
Laser excision & 95 & 32.3 \\
Diathermia excision & 134 & 45.6 \\
Knife excision & 59 & 20.1 \\
Flap technique & 6 & 2.0 \\
Surgical radicality (margins) & & \\
Yes & 254 & 86.4 \\
No & 35 & 11.9 \\
Uncertain & 5 & 1.7 \\
Lymph node dissection & & \\
Yes & 27 & 9.2 \\
No & 267 & 90.8 \\
\hline
\end{tabular}

\section{Patients and methods}

A complete geographic series of carcinomas of the vulva in FIGO stages I-IV, treated during the years 1973-2001 in the Örebro Medical Region, in the central part of Sweden, was included in a retrospective study, evaluating the extent of surgery and adjuvant radiotherapy of the inguinal regions. In all, 294 patients were included in the study (Table I). The mean age of the patients was 71 years (range 32-93 years). All histological slides were reviewed at the Department of Pathology, Örebro University Hospital. One hundred and ten tumors were in FIGO stage I (37\%), 115 tumors in stage II (39\%), 43 tumors in stage III (15\%), and 26 tumors in stage IV $(9 \%)$. One hundred and seventeen tumors were well differentiated (FIGO grade 1), 123 were moderately differentiated (FIGO grade 2), and 31 were poorly differentiated (FIGO grade 3 ). Twenty-three tumors were not graded. In 269 cases the histology was squamous cell carcinoma (92\%) (Table I). The primary surgery was total vulvectomy $(56 \%)$, partial vulvectomy (21\%), or local resection of the tumor (15\%). In 23 cases $(8 \%)$ only multiple biopsies were taken due to advanced disease. Inguinal lymph node dissection was not part of the standard surgery during this time period (Table II). The surgery was performed at three departments of Gynecology and Obstetrics, but all patients were then referred to the Department of Gynecological Oncology, Örebro University Hospital, for postoperative evaluation and treatment. The time interval between surgery and external beam radiotherapy was 4-6 weeks. The main type of radiotherapy was adjuvant inguinal irradiation. Two separate, symmetrical and rectangular inguinal fields were irradiated. Photon and electron beams were combined (50:50) and administered every second day, 5 days 

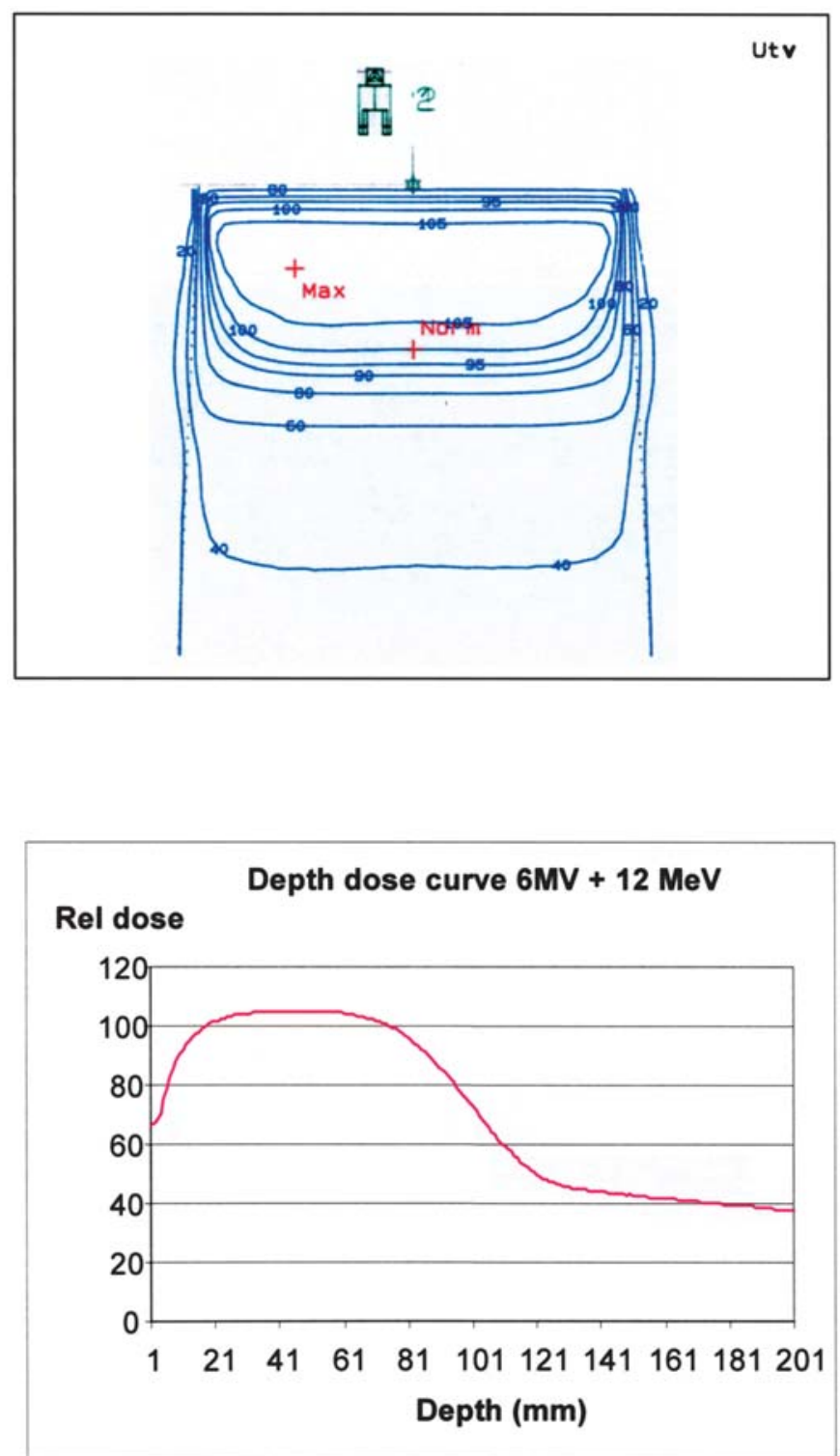

Figure 1. Dose distribution curves for a standard treatment of the inguinal region with a combination of $6 \mathrm{MV}$ photon beams and $12 \mathrm{MeV}$ electron beams. A depth dose curve illustrates the relative dose at various depths from the surface of the skin.

a week. The electron beam energy varied from 9 to $16 \mathrm{MeV}$. A standard dose distribution and depth dose curve are illustrated in Fig. 1. Three different fraction schedules were used during this period: i) 2.64 Gy given twice daily up to $47.5 \mathrm{~Gy}$, ii) 3.0 Gy given once daily up to $54.0 \mathrm{~Gy}$, and iii) $2.0 \mathrm{~Gy}$ given once daily up to $50.0 \mathrm{~Gy}$. In 23 cases vulva was also included in the fields (butterfly fields), and in 8 cases pelvic fields were used. The twice daily schedule was only used for inguinal irradiation (Table III). The time interval between the fractions was 4-6 $\mathrm{h}$ in the twice daily schedule. Most treatments were given on an outpatient basis.

All patients were followed-up for at least 10 years and no cases were lost to follow-up. The mean follow-up time was 101 months (range 19-252 months). During all visits symptoms and signs related to the therapy were recorded, the vulva and the inguinal regions were carefully inspected and pigmentation, formation of new vessels (telangiectasias), fibrosis, or lymph
Table III. Type of radiotherapy, targets and schedules used in the complete series.

\begin{tabular}{lrr}
\hline & Number & Percent \\
\hline Type of radiotherapy & & \\
$\quad$ Adjuvant & 186 & 63.3 \\
Curative & 28 & 9.5 \\
Palliative & 16 & 5.4 \\
None & 64 & 21.8 \\
Target & & \\
Inguinal lymph nodes & 199 & 67.7 \\
Vulva & 23 & 7.8 \\
Pelvis & 8 & 2.7 \\
None & 64 & \\
Schedule & & 41.8 \\
2 x 2.64 Gy x 9a & 96 & 20.0 \\
1 x 3.0 Gy x 18 & 46 & 38.3 \\
1 x 2.0 Gy x 25 & 88 & \\
\hline
\end{tabular}

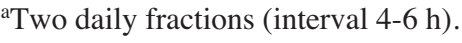

edema were recorded as well as local and regional tumor control.

The first follow-up visit was after 1 month, then every 3 months during the first year, every 4 months during the second and third years, and every 6 months up to five years and then annually up to 10 years. All data were collected in a computerized database at the Department of Gynecological Oncology, Örebro.

In the statistical analyses, survival curves were generated using the Kaplan-Meier technique and differences were tested with the chi-square or log-rank tests. The chi-square test was also used for comparison of proportions and the independent t-test for comparing means. Logistic regression analysis was used in multivariate analysis of late tissue reactions. P-values $<0.05$ were regarded as statistically significant. The Statistica (StatSoft, Inc.) software package (version 7.0, 2004) was used for the statistical analyses.

\section{Results}

In the complete series 127 recurrences $(43.2 \%)$ were recorded. Local (vulva) $(23.6 \%)$ and regional (inguinal lymph nodes) recurrences $(18.7 \%)$ were most frequent. Pelvic $(2.4 \%)$ and distant metastases $(4.4 \%)$ were rather uncommon in this series (Table IV). The median time from treatment to recurrence was 11.0 months. Type of surgery was not associated with the risk of tumor recurrence. A more limited type of surgery (local tumor excision) did not deteriorate the local tumor control in the vulva compared with extensive radical vulvectomy (Table V). The surgery was radical (tumor-free margins) in 254 cases $(86.4 \%)$, non-radical in 35 cases $(11.9 \%)$ and with uncertain surgical margins in 5 cases $(1.7 \%)$. Inguinal lymph node dissection was performed in only 27 patients $(9.2 \%)$ and this was not standard treatment in this series. 
Table IV. Sites of recurrences.

\begin{tabular}{lrr}
\hline Site & Number & Percent \\
\hline Vulva & 70 & $23.6^{\mathrm{a}}$ \\
Perineal region & 10 & $3.4^{\mathrm{a}}$ \\
Inguinal region & 55 & $18.7^{\mathrm{a}}$ \\
Pelvis & 7 & $2.4^{\mathrm{a}}$ \\
Distant metastases & 13 & $4.4^{\mathrm{a}}$ \\
Local & 59 & $46.5^{\mathrm{b}}$ \\
Regional & 40 & $31.5^{\mathrm{b}}$ \\
Distant metastases & 7 & $5.5^{\mathrm{b}}$ \\
Local + regional & 8 & $6.3^{\mathrm{b}}$ \\
Local + distant & 6 & $4.7^{\mathrm{b}}$ \\
Regional + distant & 5 & $3.9^{\mathrm{b}}$ \\
Local + regional + distant & 2 & $1.6^{\mathrm{b}}$ \\
\hline
\end{tabular}

aPercent of all included patients; ${ }^{\text {bpercent }}$ of all recurrences.

Table V. Type of surgery and local vulvar recurrences.

\begin{tabular}{lcc}
\hline Type of surgery & Number & Percent \\
\hline Radical vulvectomy & 37 & 22.4 \\
Partial vulvectomy & 18 & 28.6 \\
Local excision & 12 & 27.9 \\
& & $\mathrm{P}=0.548$ \\
\hline
\end{tabular}

Radiotherapy was administered in 230 cases $(78.2 \%)$ in total. In 186 cases $(63.3 \%)$ it was as postoperative adjuvant therapy, in 28 cases $(9.5 \%)$ as radiotherapy with curative intent for residual carcinoma, and in 16 cases $(5.4 \%)$ as palliative therapy for advanced disease. In 64 cases $(21.8 \%)$ no radiotherapy was given. The inguinal lymph nodes were irradiated in 199 cases $(67.7 \%)$, vulva was included in the radiation fields in 23 cases $(7.8 \%)$, and pelvic irradiation was used in only 8 cases $(2.7 \%)$.

The 5-year overall survival rate of the complete series was $53 \%$. In stage I the survival rate was $69 \%$ and in stage II $54 \%$ (Fig. 2). In the complete series 107 patients $(36.4 \%$ ) died due to their vulva cancer and 85 patients $(28.9 \%)$ due to other, non-cancer related, diseases. The 5-year relapsefree survival (RFS) rate was $55 \%$. In stages I and II the RFS was $60 \%$ and in stages III and IV it was $33-36 \%$ (Fig. 3). Tumor grade was significantly $(\mathrm{P}=0.007)$ associated with the relapse-free survival rate (Fig. 4). In stages I and II, type of surgery (radical vulvectomy, partial vulvectomy or local tumor excision) was not associated with the RFS rate (Fig. 5).

The inguinal relapse-free survival rate was $75 \%$ both for patients treated with adjuvant inguinal irradiation without lymphadenectomy and patients treated with primary lymphadenectomy \pm inguinal irradiation (depending on surgical lymph node status) (Fig. 6). Patients treated with primary

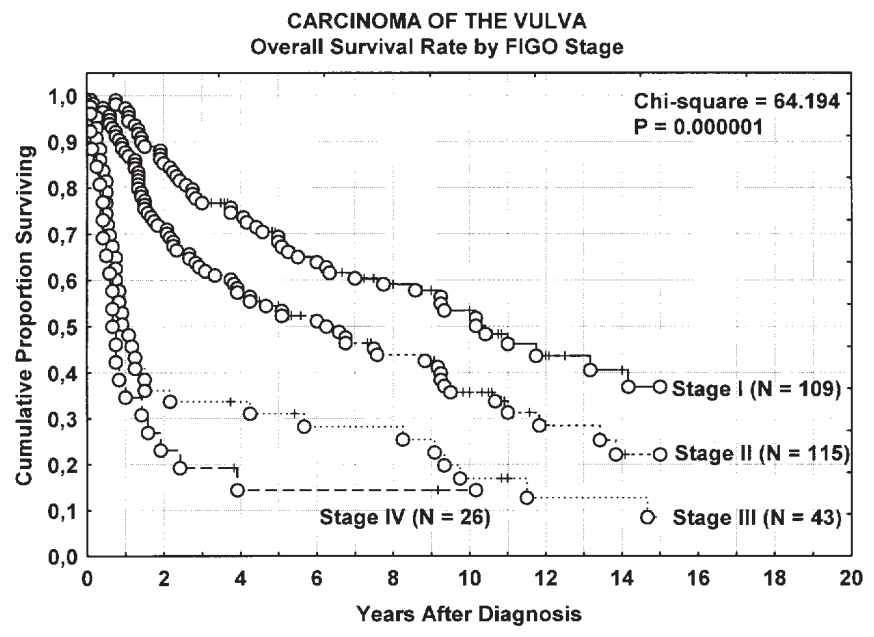

Figure 2. The overall survival rate of the complete series versus the tumor stage according to FIGO.

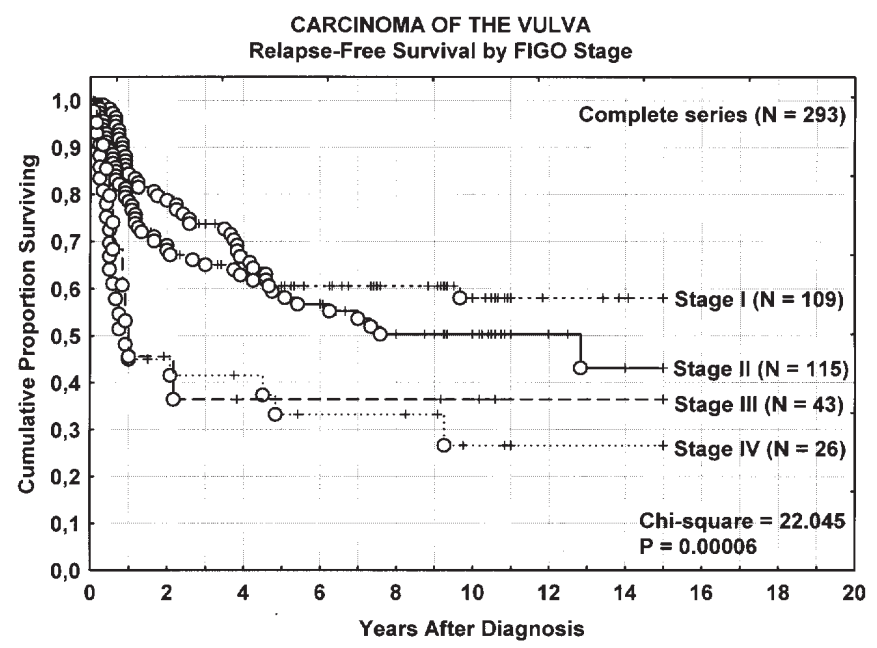

Figure 3. The relapse-free survival of the complete series versus tumor stage according to FIGO.

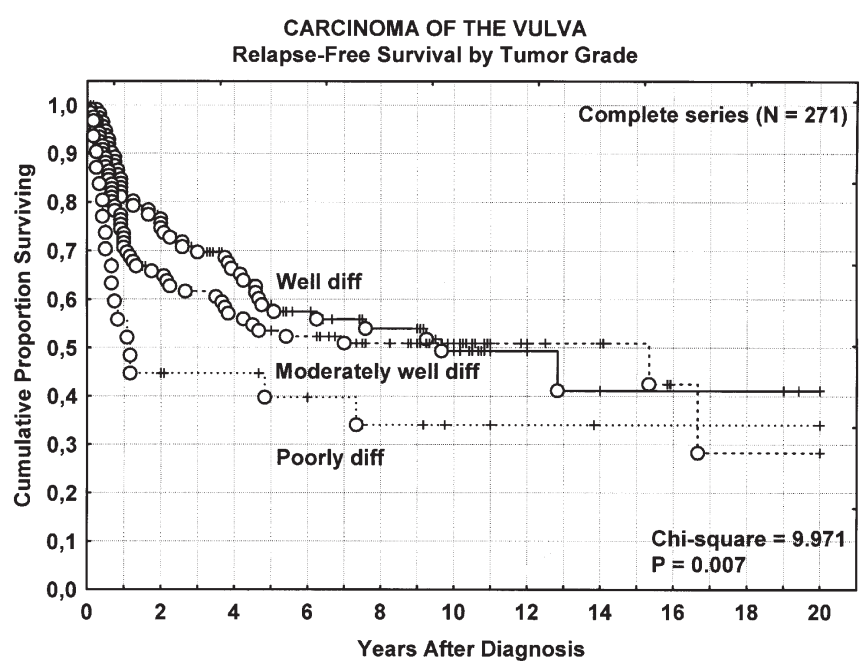

Figure 4. The relapse-free survival of the complete series versus tumor grade (well, moderately and poorly differentiated tumors). Patients with poorly differentiated carcinomas had a significantly worse prognosis compared with patients with well differentiated or moderately differentiated carcinomas. 


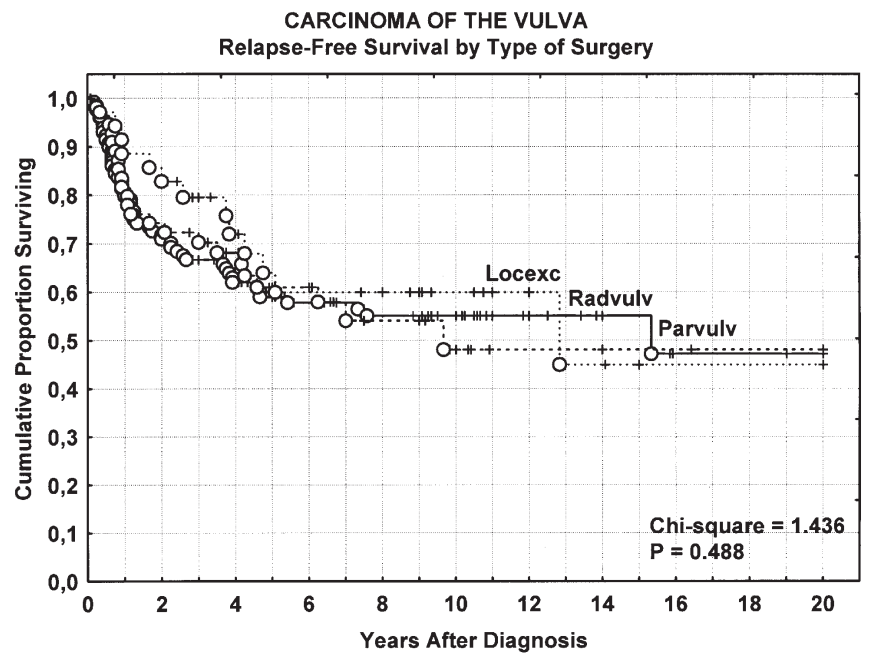

Figure 5. The relapse-free survival versus type of surgery. Radvulv, total (radical) vulvectomy; parvulv, partial vulvectomy; locexc, local excision of the tumor. More extensive surgery did not improve the survival rate.

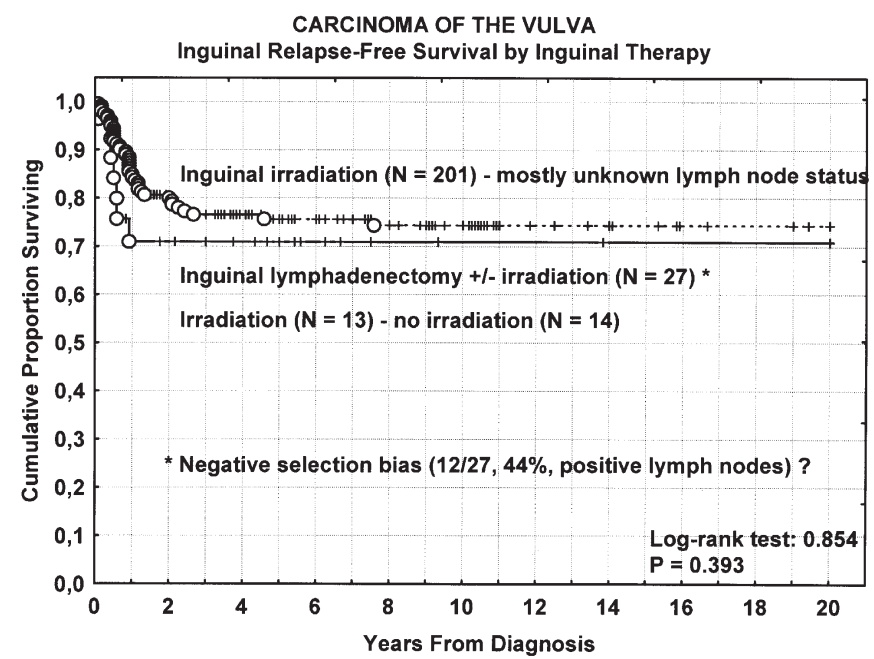

Figure 6. The inguinal relapse-free survival versus type of inguinal treatment. Inguinal irradiation (adjuvant) alone or inguinal lymphadenectomy \pm postoperative irradiation. The long-term regional tumor control was similar for the two groups.

lymphadenectomy and postoperative inguinal irradiation due to positive lymph nodes had a bad prognosis with only $21 \%$ inguinal relapse-free survival rate at 5 years. On the other hand, patients treated with primary vulvectomy and lymphadenectomy and showing negative nodes had an excellent prognosis and no inguinal recurrences were noted in this group.

Postoperative complications were recorded in 64 cases (21.8\%). Wound infection and wound rupture were recorded in $5.4 \%$ each and postoperative bleeding in $2.4 \%$. Only two cases of deep venous thrombosis occurred. Postoperative complications were significantly $(\mathrm{P}=0.027)$ more frequent in the subgroup undergoing inguinal lymphadenectomy (Table VI). Acute tissue reaction during irradiation was common, e.g. wet dermatitis in $31 \%$ of the cases. Late tissue reactions after irradiation were increased tissue pigmentation
Table VI. Surgical complications.

\begin{tabular}{|c|c|c|}
\hline & \multicolumn{2}{|c|}{ Lymph node dissection } \\
\hline & Yes & No \\
\hline \multicolumn{3}{|c|}{ Early postoperative complications } \\
\hline None & $15(56 \%)$ & $141(77 \%)$ \\
\hline Bleeding & $2(7 \%)$ & $4(2 \%)$ \\
\hline Wound infection & $7(26 \%)$ & $10(5 \%)$ \\
\hline Wound rupture & $5(19 \%)$ & $11(6 \%)$ \\
\hline \multirow[t]{2}{*}{ Thrombosis } & 0 & $2(1 \%)$ \\
\hline & & $\mathrm{P}=0.027$ \\
\hline \multicolumn{3}{|c|}{ Late postoperative complications } \\
\hline None & $20(91 \%)$ & $161(96 \%)$ \\
\hline Lymph edema & $1(7 \%)$ & 0 \\
\hline Erysipelas & 0 & $1(1 \%)$ \\
\hline Vaginal stenosis & 0 & $2(1 \%)$ \\
\hline \multirow[t]{2}{*}{ Other } & $1(5 \%)$ & $3(2 \%)$ \\
\hline & & $\mathrm{P}=0.120$ \\
\hline
\end{tabular}

Table VII. Radiation reactions.

Number Percent

$\begin{array}{lrr}\text { Early radiation reactions } & & \\ \text { Acute erytema } & 118 & 51.3 \\ \text { Wet dermatitis } & 72 & 31.3 \\ \text { Cutaneous infection } & 6 & 2.6 \\ \text { Other (diarrhea, urinary tract infection) } & 8 & 3.5 \\ \text { Late radiation reactions } & & \\ \text { Cutaneous pigmentation } & 87 & 37.8 \\ \text { Fibrosis (inguinal region) } & 63 & 27.4 \\ \text { Telangiectasia } & 17 & 7.4 \\ \text { Lymph edema } & 16^{\mathrm{a}} & 7.0 \\ \text { Thrombosis } & 2 & 0.9 \\ \text { Other } & 9 & 3.9\end{array}$

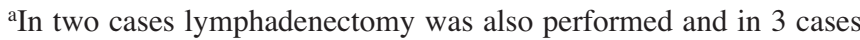
a more extended irradiation (including vulva and the pelvic region) was performed due to advanced disease.

in $38 \%$, fibrosis in $27 \%$, and new vessel formation (telangiectasia) in $7 \%$, and lymph edema in $7 \%$ (Table VII). A combination of lymphadenectomy and inguinal irradiation increased the risk of lymph edema from $6 \%$ to $17 \%$ when compared with irradiation alone. However, a logistic regression analysis showed that the increased risk of lymph edema was due to the surgical lymphadenectomy (odds ratio 4.6; $\mathrm{P}=0.033$ ) and not the radiation dose (Table VIII). Chronic lymph edemas were the most serious late tissue reactions noted after 
Table VIII. The risk of lymph edema after surgery and radiotherapy. ${ }^{\mathrm{a}}$

\begin{tabular}{lclc}
\hline Factor & Odds ratio & $95 \%$ C.I. & P-value \\
\hline Radiotherapy (D/F) & 1.00 & $0.50-2.00$ & 0.990 \\
Lymphadenectomy & 4.63 & $1.11-19.21$ & 0.033 \\
\hline
\end{tabular}

${ }^{a}$ Logistic regression analysis. D/F, dose per fraction (2.0 Gy versus 3.0 or $2.64 \mathrm{~Gy})$.

treatment with extended surgery and radiotherapy in this series of vulvar carcinomas.

\section{Discussion}

Cancer of the vulva is uncommon and represents only $4 \%$ of all gynecological malignancies. Squamous cell carcinomas normally account for $90 \%$ of the cases (2). In our series squamous cell carcinomas accounted for $92 \%$ of the tumors. The mean age was 71 years in our series which is also in agreement with the literature. In the complete series the overall 5-year survival rate was $53 \%$ and the cancer-specific survival rate was $66 \%$. Similar figures are presented in the 25 th Annual Report on the Results of Treatment in Gynecological Cancer (18). However, Manavi et al (4) were able to achieve a 5-year survival rate of 70\% in 613 cases of all FIGO stages, and Hacker (3) reported that the overall prognosis for all stages of primary squamous cell carcinomas of the vulva was good with a 5 -year survival rate of $70 \%$. These differences might be explained by various selection criteria in different series. Our study includes a complete geographic series of vulvar carcinomas including all incidental cases during a well-defined period of time. No cases were excluded or lost to follow-up. The Annual Report data were collected from 61 different institutions worldwide and included patients treated during the years 1996-1998.

In our series $83 \%$ of the relapses were local or nodal and $17 \%$ were pelvic or distant. Perez et al (9) reported that $80-95 \%$ of relapses were local or nodal. In their series $45 \%$ of the patients had recurrences after 5 years, 29\% had local vulvar recurrence, $19 \%$ had groin recurrence, $4 \%$ had pelvic recurrence, and $4 \%$ had distant metastases. Fonseca-Moutinho et al (14) reported that $31 \%$ of the patients had recurrences, $27 \%$ had local vulvar local recurrence, $13 \%$ had local and groin recurrences, $16 \%$ had groin recurrence, and 9\% had distant metastases in the fifth year. In our series the recurrence rate in the vulva was $30 \%$ and in the groin it was $14 \%$. The median time to relapse of the vulva was 21 months. According to Stehman et al (6) the median time to relapse of the vulva was 36 months and the median survival after recurrence was 52 months. The median time to relapse in the groin was 8 months in our series and 7 months in the series of Stehman et al. The median survival after recurrence in the groin was 9 months (6). The overall survival rate at 5 years in our series was $7 \%$. In a series presented by Katz et al (20) the 5-year inguinal node recurrence rate was $15 \%$, and the median survival from the time of diagnosis was only 7 months. The survival rate at 5 years in that series was $17 \%$. After lymph node dissection and radiotherapy the inguinal node recurrence rate was $11 \%$, after lymph node dissection alone it was $16 \%$, and after radiotherapy alone it was also $16 \%$. The only significant predictor of inguinal node recurrence in their series was local vulvar recurrence. After combined lymph node dissection and radiotherapy there were no significant correlations between local tumor factors and the rates of inguinal node recurrence. After lymph node dissection alone moderately and poorly differentiated tumors were at higher risk for recurrence at 5 years (19). Local vulvar recurrences are treatable by repeat excision with a control rate that is comparable to the control rate of the primary disease. Modified therapies may be associated with an increased risk of recurrence in the vulva. Patients may be at constant risk over time. Recurrence in the groin appears to represent persistent disease, occurring early and near the treated site. These recurrences are often lethal (6). The salvage rate for patients with groin recurrence is less than $10 \%$ according to the literature, but it was $14 \%$ in the group of squamous cell carcinomas in our series.

Lymph node recurrences were not significantly associated with tumor stage or total radiation dose given in our series. This is in agreement with the data presented by Perez et al (5). In our series we did not use a bolus dose over the vulva or the inguinal regions. According to Perez et al use of a bolus over the vulva was correlated with minimally improved tumor control, but in the inguinal regions there was no correlation of tumor control with the use of a bolus (5). The groin recurrence rate was 6 in $11(54.5 \%)$ cases with positive nodes after lymphadenectomy and postoperative irradiation. Thus, both surgical and radiotherapy techniques seem to have been suboptimal in this situation.

Age was a highly significant prognostic factor with regard to the cancer-specific survival rate in our series. Older age carried a reduced survival rate. Perez et al (5) found in a multivariate analysis that the age of the patient was the only significant prognostic factor.

FIGO stage was also an important prognostic factor. The majority of patients were seen in surgical stages I and II. The overall 5-year survival rate was $62 \%$ in these stages. In advanced stages III and IV 49\% experienced recurrences and the median disease-free survival was 12 months in our series. The 1-year overall survival rate was $45 \%$ in stages III-IV and $36 \%$ in stage IV. In a series presented by Fonseca-Moutinho et al (14) all patients with vulvar cancer beyond the vulva were dead within 12 months after diagnosis. Our results are comparable with the Annual Report data (40\% 12-month survival in stage IV) (18).

Lymph node metastasis is the single most important prognostic factor for survival according to the literature and presence of inguinal lymph node metastases results in a 50\% reduction in long-term survival (5). Pelvic and distant metastases are common among patients with lymph node metastases. According to Lataifeh et al (19) the probability of disease-free survival at 5 years was $67 \%$ in patients with no lymph node metastases, also $67 \%$ in patients with one node microscopically involved, but only $26 \%$ if two or more nodes were involved. Lymph node involvement was the only covariate independently influencing disease-free survival in 
their series. The overall survival probability at 5 years was $22 \%$ for patients with bilateral lymph node involvement (8). In a prospective GOG study the involvement of inguinal lymph nodes was rated as clinically negative in $81 \%$ of the cases. Of the clinically negative lymph nodes, $24 \%$ were affected by tumor according to histopathology. A falsepositive lymph node diagnosis was obtained in $20 \%$ of the cases (20). In early stages $20-30 \%$ of the patients have positive inguinal-femoral lymph node metastasis confirmed by surgery. Prognosis is better after lymphadenectomy compared to surveillance of the groin (2). Control of clinically suspicious inguinal lymph nodes is excellent after treatment with radiotherapy alone or preoperative radiotherapy according to Katz et al (20). Mariani et al (21) reported inguinal node metastases in $17 \%$ in stage I, $38 \%$ in stage II, $57 \%$ in stage III, and the overall rate was $34 \%$. Contralateral metastases were never observed with negative ipsilateral nodes. Overall survival rate was $64 \%$ in those with positive nodes treated with complementary radiotherapy. The 5-year overall survival rate was $53 \%$ in our series regardless of stage and inguinal node status. Lymph node status was primarily unknown in our series since lymphadenectomy was not routinely performed. Similar strategies were reported in the Annual Report series where the nodes were not assessed in $61 \%$ of the cases (18). In the same series the survival dropped from $69 \%$ for negative nodes to $14 \%$ for $3-4$ positive nodes. In our own series all patients with positive lymph nodes were dead within 24 months.

Tumor size greater than $4 \mathrm{~cm}$ or $2 \mathrm{~cm}$ was not associated with higher local recurrence rate in our series. The local recurrence rate was $13 \%$ for tumors $>4 \mathrm{~cm}$ and $24 \%$ for tumors $<4 \mathrm{~cm}$. In the GOG protocol No. 36, on the other hand, tumor size $>4 \mathrm{~cm}$ was a significant risk factor for recurrence in the vulva (5). Tumor size of $2 \mathrm{~cm}$ was the most effective cut-off limit for survival prognosis according to Shimm et al (15). Frumovitz et al (22) did not find any association between tumor size and local recurrence rate. Lesion diameters of $1 \mathrm{~cm}$, $2 \mathrm{~cm}$, or $3 \mathrm{~cm}$ have been proposed as limits for conservative resection with or without inguinal node dissection.

Sedlis et al (23) proposed that tumor thickness is a more reproducible measurement than depth of invasion (24). Tumor thickness $>5 \mathrm{~mm}$ has been correlated with a higher incidence of local relapse (5). The association of relapses with tumor thickness (not measured) is unknown in our series.

Lymphatic space involvement was associated with a local recurrence rate of $25 \%$ and cases with no space involvement $17 \%$ (non-significant) in our series. In a series presented by Perez et al lymphatic space involvement and perineural growth were significant risk factors for local recurrence $(5,25)$. Lymph-vascular space invasion has also been associated with inguinal node metastases and poor prognosis (24).

The tumor-free margins are the most important predictive factor for local recurrence rate $(2,16)$. The local recurrence rate was $50 \%$ if margins were $<8 \mathrm{~mm}$ (16). De Hullu showed no local recurrence if margins were $>8 \mathrm{~mm}$, but $23 \%$ if margins were $<8 \mathrm{~mm}$. A statistically increased risk of local recurrence was noted for patients with a pathologic tumor-free margin of $\leq 8 \mathrm{~mm}$ (16). The distribution of recurrences appeared to be minimally influenced by the radicality of therapy; it is the closest margin of excision that determines local recurrence
(14). In $50 \%$ of the patients the tumor-free margins were $<8 \mathrm{~mm}$ in spite of a macroscopic intention of $1 \mathrm{~cm}$. Therefore, the authors $(2,16)$ advise wide local excision with an intentional surgical margin of $2 \mathrm{~cm}$. In our series local (vulvar) recurrences occurred in $24 \%$ in cases with margins $\leq 8 \mathrm{~mm}$, and in $20 \%$ in cases with margins $>8 \mathrm{~mm}$ (non-significant). Nor was $10 \mathrm{~mm}$ a significant cut off margin in our series.

Present treatment of vulvar carcinoma is wide local excision with unilateral or bilateral inguinofemoral lymphadenectomy via the triple incision technique. Postoperative radiotherapy is given in the case of more than one intranodal metastasis or one lymph node metastasis with extra nodal growth (2). It is now recommended to perform a full inguinofemoral lymphadenectomy (medial femoral nodes and all superficial lymph nodes) (26). Preti et al found an increased risk of relapse after the triple incision (14), but Hacker et al showed that survival with the triple incision technique was similar to that in historical controls that had en block radical surgery (14). Omission of lymphadenectomy is regarded to be safe in squamous cell carcinomas with a depth of invasion $\leq 1 \mathrm{~mm}$ (2). Contralateral groin dissection should be performed only when the ipsilateral inguinal nodes are involved.

Irradiation to doses of $50 \mathrm{~Gy}$ is an effective alternative to electively treat the regional nodes (27). If palpable inguinal lymph nodes are present, a lymphadenectomy is preferred, followed by 50 Gy to the inguinal-femoral and pelvic nodes. If irradiation alone is used, additional doses are delivered with reduced fields to a total of 65-70 Gy (5). In stage IA cancer of the vulva wide local excision is an adequate therapy. Routine groin lymphadenectomy is unwarranted. No pathologic or clinical risk factors have been identified for vulvar recurrence in early carcinoma of the vulva after complete surgical resection. Surgical radicality was not associated with recurrence rate (28). There was no significant impact of surgical technique on disease-specific and overall survival. In a multivariate analysis, type of surgical treatment was an independent predictor of vulvar recurrence, but not for inguinal or pelvic recurrence. Extra capsular disease was the only independent predictor for the risk of inguinal and pelvic recurrences $(H R=4.19)$. No single variable was able to predict the risk of distant metastases (29). In our series $14 \%$ regional recurrences occurred in surgically negative groins, whereas failure occurred in $40 \%$ if the nodes ( $\geq 1$ node) were positive at surgery. In all, in $25 \%$ of the cases regional recurrences occurred in patients who underwent groin dissection.

In our series recurrence-free, disease-specific, and overall survival rates were not significantly associated with the surgical technique (total vulvectomy vs. partial vulvectomy or wide local excision) after correction for tumor stage and tumor size. The surgical technique was not a significant predictor of vulvar, inguinal, pelvic or distant recurrences.

The GOG study showed that radical surgical intervention resulted in chronic lymphatic edema of the lower extremity in $27 \%$ of the cases (20).

Podratz et al (30) reported that $69 \%$ of the patients treated by lymphadenectomy had lymphatic edema and $85 \%$ had wound separation. Considering that $75 \%$ of removed lymph nodes are tumor free, such a high level of morbidity is unacceptable (4). In our series of 27 cases that underwent inguinal lymphadenectomy only one case of leg lymph 
edema was recorded. In the complete series two cases of leg lymph edema and 21 cases (8\%) with wound separation were recorded.

The results of palpation in predicting the lymph node status are disappointing. In clinically negative groins $22 \%$ had occult lymph node metastases (7). PET is useless, CT is probably of little value, and the role of MRI is uncertain $(2,31,32)$. Ultrasound-guided fine-needle biopsy is a promising technique $(33,34)$. There might be a role for preoperative detection of lymph node metastases, leading to direct complete inguinalfemoral lymphadenectomy instead of the sentinel lymph node procedure (2). Malignant nodes tend to develop a rounded shape, and a ratio of less than 2 for the long and short axis diameter indicates malignancy (ultrasound). The sensitivity was $82 \%$ and the specificity was $87 \%$ (35). The sensitivity for the combined technique (ultrasound plus fine-needle biopsy) was $93 \%$ (34). The identification rate of the sentinel node technique is nearly $100 \%$ with preoperative lymphoscintigram and probe-guided surgery. An extensive study is taking place in the USA (GOG-173) to determine the diagnostic sensitivity of the sentinel node procedure in vulvar cancer. The negative predictive value of a negative sentinel node is $100 \%$ (2). Patients with positive sentinel nodes should be randomly assigned between complete inguinal-femoral lymphadenectomy and primary radiation to prevent as far as possible the impressive morbidity of a complete inguinal-femoral lymphadenectomy (2).

Irradiation of N0 or N1 inguinal-femoral nodes is an alternative to lymphadenectomy for squamous cell carcinoma of the vulva if proper irradiation technique and dose are used. The acute and late morbidity are less than with lymphadenectomy (10). In patients with metastatic deep inguinalfemoral lymph nodes at lymphadenectomy, postoperative irradiation should be given to this region as well as to the pelvis (20). Inguinal lymph node relapses occurred in 5\% of patients with groin radiation and in $10 \%$ of patients without irradiation in a non-randomized series of 135 patients with T1, N0-1 tumors treated between 1974 and 1990 (Vienna, Austria) (4). In our series inguinal node relapses occurred in 15 of 110 patients (14\%) in stage I. No case of leg edema or thrombosis was recorded among patients who only received radiologic treatment. In combined treatment leg edema is probably mainly an effect of surgery (36). In 34 patients with a 'low-risk' profile no surgery and no radiotherapy were applied to inguinal regions and the rate of recurrence was very low $(3 \%)$.

We presume that the omission of groin radiation seems to be justified in low-risk cases in the T1, N0-1 stage (no central location, no vessel invasion, tumor thickness $\leq 2 \mathrm{~mm}$, and G1, G2 tumors) (4). In our group of low-risk cases the inguinal node recurrence rate was $3 \%$ after no surgery or radiotherapy. Therefore, the omission of groin radiation seems justified in this group. This low-risk group is probably not comparable with the group defined by Manavi et al, since vessel space invasion and tumor thickness are not evaluable, and 14 of 34 tumors had a central location (clitoris, urethra, and perineum).

Some investigators have suggested treating patients with clinically negative groins with wide local excision of the vulva combined with radiotherapy of the groin only. The use of elective groin irradiation in patients with vulvar cancer is controversial (2). In our series inguinal irradiation seems to be an option comparable with lymphadenectomy in patients with clinically negative lymph node status. The 5-year inguinal relapse-free survival was similar $(75 \%)$ for both treatment strategies. In 1992, Stehman et al (6) published GOG study 88. The objective of that study was to document the rates of recurrence between patients randomly assigned to either groin dissection or radiation to the intact groin. Five out of 27 patients $(18.5 \%)$ who received primary groin radiation developed a groin recurrence, which was regarded as an unacceptably high failure rate, and therefore the study was closed in advance. In our series inguinal node relapses occurred in 15 of 110 patients (14\%) in stage I. This is a high failure rate and comparable to that of the GOG \# 88 study. Petereit et al (10) showed good local control and survival after either inguinal-femoral lymphadenectomy or elective radiotherapy. A Cochrane review in 2002 stated that primary radiotherapy to the groin results in less morbidity but also in a higher number of groin recurrences compared with surgery (11).

The addition of radiotherapy to inguinal-femoral lymphadenectomy can worsen the already serious side effects of this procedure (2). In our series a combination of surgery (lymphadenectomy) and inguinal irradiation increased the risk of lymph edema compared with irradiation alone.

A large single institution series from M.D. Anderson showed a 5 -year groin recurrence rate of $15 \%$. The recurrence rate was similar after superficial inguinal-femoral lymphadenectomy alone (17\%), radiotherapy alone or a combination. The radiation fields were unusually narrow and many recurrences occurred outside of them (19).

In our series lymphadenectomy \pm irradiation resulted in 6 inguinal recurrences of $24(25 \%)$ treated patients and inguinal (or none in selected low-risk cases) irradiation in 43 inguinal recurrences among 245 patients (18\%). Clinical low-risk cases and lymph node negative cases after lymphadenectomy do well and do not seem to need any irradiation. In clinical medium- or high-risk cases not suitable for surgery and with clinical negative groins addition of prophylactic postoperative irradiation only gives a moderate protection from recurrences, since the recurrence rate is still $20 \%$ in this group.

In conclusion, prospective randomized studies are necessary to determine whether primary radiotherapy with an adequate radiation dose and technique is a safe treatment of the groin in a selection of patients with early-stage vulvar cancer (2). The radiotherapy technique must be improved and the individual treatment planning seems to be of utmost importance. The value of chemoradiation needs also to be tested in controlled clinical trials (27).

\section{References}

1. Cancer Incidence in Sweden 2003. Swedish Cancer Registry, Centre for Epidemiology. The National Board of Health and Welfare, Stockholm, Sweden, 2004. ISBN 91-7201-915-8.

2. de Hullu JA, Oonk MHM and van der Zee AGJ: Modern management of vulvar cancer. Curr Opin Obstet Gynecol 16: 65-72, 2004.

3. Hacker NF: Vulvar cancer. In: Practical Gynecologic Oncology. Berek JS and Hacker NF (eds). 3rd edition. Williams and Wilkins, Baltimore, pp553-596, 2000. 
4. Manavi M, Berger A, Kucera E, Vavra N and Kucera H: Does T1, N0-1 vulvar cancer treated by vulvectomy but not lymphadenectomy need inguinofemoral radiation? Int J Radiat Oncol Biol Phys 38: 749-753, 1997.

5. Perez CA, Grigsby PW, Clifford Chao KS, Galakatos A, Garipagaoglu M, Mutch D and Lockett MA: Irradiation in carcinoma of the vulva: Factors affecting outcome. Int J Radiat Oncol Biol Phys 42: 335-344, 1998.

6. Stehman FB, Bundy BN, Thomas G, Varia M, Okagaki T, Roberts J, Bell J and Heller PB: Groin dissection versus groin radiation in carcinoma of the vulva: A Gynecologic Oncology Group study. Int J Radiat Oncol Biol Phys 24: 389-396, 1992.

7. Gonzalez Bosquet JG, Kinney WK, Russell AH, et al: Risk of occult inguinofemoral lymph node metastasis from squamous carcinoma of the vulva. Int J Radiat Oncol Biol Phys 57: 419-424, 2003.

8. Homesley HD, Bundy BN, Sedlis A, Yordan E, Berek JS, Jahshan A and Mortel R: Prognostic factors for groin node metastasis in squamous cell carcinoma of the vulva (A Gynecologic Oncology Group Study). Gynecol Oncol 49: 279-283, 1993.

9. Perez CA, Grigsby PW, Galakatos A, Swanson R, Camel HM, Kao M-S and Lockett MA: Radiation therapy in management of carcinoma of the vulva with emphasis on conservation therapy. Cancer 71: 3707-3716, 1993.

10. Petereit DG, Mehta MP, Buchler DA and Kinsella TJ: Inguinofemoral radiation of N0, N1 vulvar cancer may be equivalent to lymphadenectomy if proper radiation technique is used. Int J Radiat Oncol Biol Phys 27: 963-967, 1993.

11. Van der Velden J and Ansink A: Primary groin irradiation versus primary groin surgery for early vulvar cancer (Cochrane Review). In: The Cochrane Library, Issue 1. Oxford, Update Software, 2002

12. Homesley HD, Bundy BN, Sedlis A, Yordan E, Jahshan A and Mortel R: Assessment of current International Federation of Gynecology and Obstetrics staging of vulva carcinoma relative to prognostic factors for survival (A Gynecologic Oncology Group Study). Am J Obstet Gynecol 164: 997-1004, 1991.

13. Gordinier ME, Malpica A, Burke TW, Bodurka DC, Wolf JK, Jhingran A, Ramirez PT and Levenback C: Groin recurrence in patients with vulvar cancer with negative nodes on superficial inguinal lymphadenectomy. Gynecol Oncol 90: 625-628, 2003.

14. Fonseca-Moutinho JA, Coelho MC and Silva DP: Vulvar squamous cell carcinoma. Prognostic factors for local recurrence after primary en bloc radical vulvectomy and bilateral groin dissection. J Reprod Med 45: 672-678, 2000

15. Shimm DS, Fuller AF, Orlow EL, Dosoretz DE and Aristizabal SA: Prognostic variables in the treatment of squamous cell carcinoma of the vulva. Gynecol Oncol 24: 343-358, 1986

16. Heaps JM, Fu YS, Montz FJ, et al: Surgical-pathologic variables predictive of local recurrence in squamous cell carcinoma of the vulva. Gynecol Oncol 38: 309-314, 1990.

17. Ansink AC, Sie-Go DMDS, van der Velden J, et al: Identification of sentinel lymph nodes in vulvar carcinoma patients with the aid of a patent blue V injection. Cancer 86: 652-656, 1999.

18. 25th Annual Report on the Results of Treatment in Gynecological Cancer. Int J Gynecol Obstet 83 (Suppl 1): 19, 2003

19. Lataifeh I, Nascimento MC, Nicklin JL, Perrin LC, Crandon AJ and Obermair A: Patterns of recurrence and disease-free survival in advanced squamous cell carcinoma of the vulva. Gynecol Oncol 95: 701-705, 2004.

20. Katz A, Eifel PJ, Jhingran A and Levenback CF: The role of radiation therapy in preventing regional recurrences of invasive squamous cell carcinoma of the vulva. Int J Radiat Oncol Biol Phys 57: 409-418, 2003.
21. Mariani L, Lombardi A, Atlante M and Atlante G: Radiotherapy for vulvar carcinoma with positive inguinal nodes. J Reprod Med 38: 429-436, 1993.

22. Frumovitz M, Ramirez PT, Tortolero-Luna G, Malpica A, Eifel P, Burke TW and Levenback C: Characteristics of recurrence in patients who underwent lymphatic mapping for vulvar cancer. Gynecol Oncol 92: 205-210, 2004.

23. Sedlis A, Homesley H, Bundy BN, Marshall R, Yordan E, Hacker N, Lee JH and Whitney C: Positive groin lymph nodes in superficial squamous cell vulvar cancer. Am J Obstet Gynecol 156: 1159-1164, 1987.

24. Burke TW, Stringer CA, Gershenson DM, Edwards CL, Morris M and Wharton JT: Radical wide excision and selective inguinal node dissection for squamous cell carcinoma of the vulva. Gynecol Oncol 38: 328-332, 1990

25. Rowley KC, Gallion HH, Donaldson ES, van Nagell JR, Higgins RV, Powell DE, Kryscio RJ and Pavlik EJ: Prognostic factors in early vulvar cancer. Gynecol Oncol 31: 43-49, 1988

26. Levenback C, Morris M, Burke TW, et al: Groin dissection practices among gynaecologic oncologists treating early vulvar cancer. Gynecol Oncol 62: 73-77, 1996.

27. Thomas GM, Dembo AJ, Bryson SCP, Osborne R and DePetrillo AD: Changing concepts in the management of vulvar cancer. Gynecol Oncol 42: 9-21, 1991.

28. Magrina JF, Gonzalez-Bosquet J, Weaver AL, Gaffey TA, Leslie KO, Webb MJ and Podratz KC: Squamous cell carcinoma of the vulva stage IA: Long-term results. Gynecol Oncol 76 : 24-27, 2000

29. Van der Velden J, Schilthuis MS, Hyde SE, ten Kate FJW and Burger MPM: Squamous cell cancer of the vulva with occult lymph node metastases in the groin: the impact of surgical technique on recurrence pattern and survival. Int J Gynecol Cancer 14: 633-638, 2004.

30. Podratz KC, Symmonds RE, Taylor WE and Williams TJ: Carcinoma of the vulva: Analysis of treatment and survival. Obstet Gynecol 61: 63-74, 1983.

31. Sohaib SA, Richards PS, Ind T, et al: MR imaging of carcinoma of the vulva. Am J Roentgenol 178: 373-377, 2002.

32. Hawnaur JM, Reynolds K, Wilson G, et al: Identification of inguinal lymph node metastases from vulval carcinoma by magnetic resonance imaging: in initial report. Clin Radiol 57: 995-1000, 2002

33. Abang Mohammed DK, Uberoi R, de B Lopes A and Monaghan JM: Inguinal node status by ultrasound in vulvar cancer. Gynecol Oncol 77: 93-96, 2000.

34. Hall TB, Barton DPJ, Trott PA, et al: The role of ultrasoundguided cytology of groin lymph nodes in the management of squamous cell carcinoma of the vulva: 5 -year experience in 44 patients. Clin Radiol 58: 367-371, 2003.

35. Mäkelä PJ, Leminen A, Kääriäinen $M$ and Lehtovirta $P$. Pretreatment sonographic evaluation of inguinal lymph nodes in patients with vulvar malignancy. J Ultrasound Med 5: 255-258, 1993.

36. Simonsen E, Nordberg U-B, Johnsson J-E, Lamm L-L and Tropé C: Radiation therapy and surgery in the treatment of regional lymph nodes in squamous cell carcinoma of the vulva. Acta Radiol Oncol 23: 433-442, 1984. 